\title{
A partire da Cristo, Redentore dell'uomo. Sant'Agostino e il Beato Giovanni Paolo II - alcuni punti di riferimento
}

\author{
Starting from Christ, the Redeemer of Man to St. Augustine \\ and Blessed John Paul II - a Few Points of Reference
}

\section{Abstract}

In Jesus Christ, the human person finds greatness, dignity and worth of humanity. This article reflects on the doctrine of the great African bishop, St. Augustine of Hippo and Pope John Paul II. It discusses the truth about Jesus Christ, the Redeemer of humankind. The concept of redemption as represented by the bishop of Hippo is in essence sacerdotal and sacrificial as it is ultimately expressed by the sacrifice of the Cross. The Cross of Jesus Christ manifests the great and merciful willingness of God towards the fallen human person who is the slave of sin. The triple victory of Christ: over sin, death and Satan, restores the human person to life in the communion with God.

\section{Keywords}

Christ the Redeemer, redemption, salvation, St. Augustine, Pope John Paul II.

\section{Introduzione}

La redenzione compiuta da Cristo svolge il ruolo di filo conduttore nelle riflessioni teologiche in ogni epoca di tempo. L'accostamento dei due Autori, il vescovo d'Ippona, e il vescovo di Roma e il papa Giovanni Paolo II, così distanti dal punto di vista storico, non è del tutto casuale. Sant'Agostino il vescovo d'Ippona è presente nella vita della Chiesa e nella mente e nella cultura di tutto 
l'Occidente, già dalla sua morte. In occasione del XVI centenario della sua conversione il papa Giovanni Paolo II ha pubblicato la sua Lettera apostolica Augustinum Hipponensem ${ }^{1}$, che rappresenta il piú completo ed autorevole quadro del pensiero su quel grande Padre e Vescovo della Chiesa che, pur dopo sedici secoli, è sempre vivo ed attuale nel pensiero della Chiesa. Nella storia della Chiesa non ci sono personaggi che siano meglio conosciuti o che abbiano esercitato nella Chiesa fino ad oggi un'influenza piú profonda come quella di Agostino. Giovanni Paulo II nella sua Lettera esprime la sua grande ammirazione e la sua stima verso questo grande Padre dell' antichità cristiana. In quanto lo scopo di questa Lettera è quello di avvicinare all'uomo del XX secolo un Padre del secolo V, lo stesso vale anche per l'uomo del XXI secolo.

Il tema che ha inspirato il presente articolo è il tema della redenzione in Cristo, di Cristo, Redentore dell'uomo, il tema densamente presente nella riflessione e nella dottrina sia del grande africano come del papa Giovanni Paolo II. La qualifica Redentore riferita a Cristo, sta ad indicare che la redenzione è opera e dono Suo. Il papa Giovanni Paolo II avendo in mente il ruolo che Cristo Redentore ha compiuto nella vita di Agostino, in particolare dal momento della sua conversione, sottolinea da parte sua: „Su questa soluzione ho inteso il bisogno di richiamare anch'io l'attenzione dei figli della Chiesa e di tutti gli uomini di buona volontà nella mia prima enciclica, appunto la Redemptor hominis, lieto di raccogliere nella mia voce la voce di tutta la tradizione cristiana" ${ }^{2}$. Questa vicinanza impone da sé la domanda, quale sia il contributo del vescovo d'Ippona nella tradizione cristiana ${ }^{3}$, e quali siano le tematiche ed i punti in comune dell'enciclica Redemptor hominis ${ }^{4}$ nel capire e nell'esprimere la fede intorno al Cristo, Redentore dell'uomo?

\footnotetext{
${ }^{1}$ Giovanni Paolo II, Lettera apostolica Augustinum Hipponensem del Sommo Pontefice Giovanni Paolo II nel XVI centenario della conversione di S. Agostino (28.08.1989; abbr. AH), in: R. Piccolomini, N. Monopoli, L'attualità di Agostino. Commento alla Lettera apostolica Agostino d'Ippona di Giovanni Paolo II, Roma 1986, p. $213-249$ [testo orig. in: AAS 79 (1987) 137-170].

${ }^{2}$ Giovanni Paolo II, AH, 18.

${ }^{3}$ Nella presentazione del pensiero del grande vescovo d'Ippona ci limitiamo alla sua celebre opera Esposizioni sui Salmi (Enarrationes in Psalmos), che rappresenta una miniera preziosa del suo insegnamento. Il testo delle Esposizioni sui Salmi viene citato secondo la traduzione che si trova nell'Opera Omnia di Sant'Agostino in edizione latino-italiana, a cura della Nuova Biblioteca Agostiniana (NBA 25-28/2).

${ }^{4}$ Giovanni Paolo II, Lettera enciclica Redemptor hominis (4 marzo 1979): AAS 71 (1979) 257324 (abbr. RH).
} 


\section{Totus Deus et totus homo}

L'interesse preminente insieme per Cristo ${ }^{5}$ eperl'uomo nel pensiero agostiniano rivela tutta la sua intrinseca dinamicità nel mistero della Redenzione. Essa confrontata con l'opera della creazione riceve il significato d'una nuova creazione dove Cristo in confronto al primo uomo diventa il nuovo Adamo. L'intervento di Cristo Salvatore e Redentore, che risale oltre quello dell'azione creatrice portandola al suo compimento, rivela tutta la particolarità dell'eterno disegno di Dio uno e trino riguardo agli uomini, della sua sconfinata misericordia ${ }^{6}$. Il punto culminante di questo disegno consiste, secondo la tradizione predominante in Occidente, nel

${ }^{5}$ La presentazione del mistero di Cristo da parte del vesovo d'Ippona è insieme filosofica e teologica, ontologica e soteriologica. Secondo l'etimologia agostiniana ancorata ai canoni tradizionali Gesù, il nome che da bambino aveva succhiato col latte materno, significa "Salvatore". In maniera riassuntiva ci viene spiegato: „I nomi di Gesù e quello di Cristo hanno due diversi significati. Gesù Cristo, nostro Salvatore, è evidentemente una sola persona, tuttavia il nome che gli è proprio è quello di Gesù. Come Elia, Mosè ed Abramo ebbero un loro proprio nome, così il Signore nostro ha un suo nome proprio, Gesù. Invece il nome di Cristo designa una funzione di carattere sacro. Lo stesso si verifica per i nomi di profeti e di sacerdoti. Cristo significa: unto, colui per mezzo del quale si sarebbe attuata la redenzione di tutto il popolo d'Israele", Agostino, Commento alla Prima Epistola di S. Giovanni 3, 6 (NBA 24/2, p. 2000). Tra i principali errori cristologici che in diverse maniere toccavano la natura teandrica di Cristo nell'epoca agostiniana sono quelli di: ebioniti (Gesù Cristo è un uomo così giusto che lo si può chiamare Figlio di Dio); docetisti (il Figlio di Dio non ha assunto un vero uomo); apollinaristi (l'uomo Gesù fu privo della mente umana); adozionisti (Gesù è stato assunto dall'eterna Sapienza come gli altri uomini sapienti con la differenza di essere nato da una Vergine); ariani (Cristo è un essere soprannaturale che dall'uomo ha preso solo il corpo); catafrigi (il Figlio di Dio non è nato da una donna, perché se bisognava mostrarsi agli occhi degli uomini, poté assumere un corpo, così, come lo Spirito Santo apparve nella colomba); manichei (Gesù Cristo è il Figlio di Dio e non il Verbo incarnato). Cf. G. Madec, Christus, in: Augustinus-Lexikon, ed. C. Mayer, vol. I, Basel 1986, p. 870.

${ }^{6}$ „In che senso dunque il disegno del Signore resta in eterno, se non perché ha già saputo e predestinato in anticipo riguardo a noi (cf. Ef 1,4)? Chi può negare la predestinazione di Dio? Prima della creazione del mondo ci ha visti, ci ha creati, ci ha corretti, ha mandato [il Figlio suo] a noi, ci ha redenti; questo è il suo disegno che resta in eterno, questo è il suo pensiero che rimane nei secoli dei secoli”, Agostino, Espos. sul s. 32, II, s. 2, 14 (NBA 25, p. 595-597). Non è possibile la riconciliazione con Dio se non attraverso la fede incentrata in Gesù Cristo: „Nessuno infatti senza questa fede che fa centro in Gesù Cristo, sia prima che dopo la sua incarnazione, si è riconciliato con Dio. È una verità questa definita in termini esattissimi dall'Apostolo: C'è infatti un Dio solo, e uno solo è il Mediatore tra Dio e gli uomini, Gesù Cristo uomo (1 Tm 2, 5)", Idem, Espos. sul s. 104, 10 (NBA 27/1, p. 798). Partendo dalla miseria del peccato è inevitabile il riferimento alla misericordia di Dio che fa da sottofondo a tutta l'economia di salvezza: „Ma ciò che l'uomo non può fare, anche se lo vuole, lo può e vuole fare Dio e l'ha fatto. Dio, nella sua infinita misericordia, ha inviato all'umanità decaduta e schiava del peccato il suo Figlio unigenito per redimerla e per liberarla", B. Mondin, L'uomo secondo il disegno di Dio. Trattato di antropologia teologica, Bologna 1992, p. 200. Intanto si deve notare che: „Cette miséricorde culmine dans le sacrifice du médiateur. C'est pourquoi la doctrine de la médiation est inséparable du sacerdoce et du sacrifice du Christ", G. Remy, Le Christ mediateur dans l'oeuvre de Saint Augustin, Lille-Paris 1979, p. 476. 
sacrificio di Cristo ontologicamente fondato sulla sua incarnazione. Il Figlio di Dio, venendo per la misericordia sulla terra, ha preso quaggiú ciò che quaggiú abbonda e ha fatto uno scambio. Ha recato molti beni all'uomo e in contraccambio ha subito molti mali, non escludendo neanche quello della morte. Si è rivestito di ciò che era necessario per redimere l'uomo perchè anche questi si rivestisse di ciò che gli consentisse, dopo esser stato riconciliato con Dio, di vivere con Lui in quanto suo figlio adottivo. Cosí si è compiuta la mirabile commutazione, si è effettuato il divino commercio ${ }^{7}$. Il Cristo è divenuto in tutto simile agli uomini eccetto il peccato, per poter, attraverso la solidarietà che lo unisce con tutto il genere umano, espiare i peccati degli uomini in nome di questa solidarietà. Egli, in quanto uomo giusto, ha propiziato Dio per l'uomo peccatore. Il valore oggettivo della redenzione di Cristo, nuovo Adamo, viene completato da sant'Agostino con la sua dimensione morale. La dimensione sacrificale della morte di Cristo su cui il vescovo d'Ippona mette l'accento particolare, lo porta a concepirla come l'atto di redenzione che necessariamente si riferisce a tutte le forze del male menzionate dalla Scrittura. La morte di Cristo è una vera ed inesorabile sorgente dei benefici (beneficia divina) che vengono concessi ugualmente a tutti gli uomini.

Prendendo in considerazione tutta questa ricchezza della tradizione cristiana riguardo alla redenzione di Cristo in gran parte fondata su sant'Agostino, il papa Giovanni Paolo II apre il suo primo documento ufficiale con le parole „Il Redentore dell'uomo, Gesù Cristo, è centro del cosmo e della storia" (RH 1). Il Pontefice nan ha nessun dubbio che dal momento in cui Dio è diventato uomo, ogni essere umano deve essere sottomesso a Cristo, e per mezzo di Lui a Dio. Secondo l'insondabile e imprescrutabile disegno di Dio „Cristo, Redentore del mondo, è Colui che è penetrato, in modo unico e irrepetibile, nel mistero dell'uomo ed è entrato nel suo «cuore»" (RH 8). Gesù nell'ora

\footnotetext{
${ }^{7}$ Cristo divenne partecipe della mortalità dell'uomo per renderlo partecipe della sua divinità: „Purtuttavia, poiché si è degnato di assumere la forma di servo e in essa si è rivestito di noi, come non ha disdegnato di assumerci in sé, cosí non ha neppure sdegnato di trasfigurare noi in sé e di parlare con le nostre parole, affinché anche noi potessimo parlare con le parole di Lui. Si è infatti compiuta questa mirabile commutazione, ha avuto luogo il divino commercio ed è stato celebrato in questo mondo dal celeste negoziatore lo scambio delle sostanze", Agostino, Espos. sul s. 30, II, s. 1, 3 (NBA 25, p. 443). Cf. Idem, Espos. sul s. 121, 5 (NBA 28/1, p. 13); Espos. sul s. 134, 5 (NBA 28/1, p. 345). Trattando degli inizi di quest'idea N. Cipriani indicando Melitone, il vescovo di Sardi in Lidia, afferma: „L'idea, che il Nuovo Testamento vede anticipata nella figura dal profeta Isaia nei Canti del Servo di JHWH, era stata fatta oggetto di meditazione già dai Padri del II secolo", Gesù il Figlio. Testi dei Padri della Chiesa, Roma 1997, p. 15. Cf. É. Bailleux, La sotériologie de saint Augustin dans le "De Trinitate", "Mélanges de Science Religieuse" 23 (1966), p. 3-4.156; G. Bonner, The desir for God and the need for grace in augustine's theology, in: Congresso Internazionale su s. Agostino nel XVI centenario della conversione, (Roma 15-20 sett. 1986), vol. I, Roma 1987, p. 205.
} 
della sua morte è passato dalla condizione concreta dell'uomo, debole, fragile e sottomessa alla morte, alla pienezza di vita di Dio e questo grazie ad un amore portato fino all'estremo.

\section{Cristo Redentore dell'uomo}

L'Occidente cristiano a cavallo dei secoli IV e V non fu esente dalle antiche obiezioni di alcuni pensatori sulla possibile salvezza anche senza Cristo, e perciò il vescovo d'Ippona ha riflettuto molto sull'annuncio scritturistico dell'opera redentrice di Cristo, privilegiandone alcuni aspetti tipici per la sua epoca. L'intero agire salvifico di Dio che per i Padri greci significava un lungo processo di tipo educativo realizzatosi mediante gli eventi storici che nel Figlio di Dio fatto uomo trovano il loro incoronamento, con lo scopo di ripristinare l'immagine di Dio nell'uomo sfigurata dal peccato (“divinizzazione dell'uomo"), per i Padri latini, i quali dettero maggiore importanza al singolo e alla sua colpa, significava, prima di tutto, il perdono del peccato comunicatogli da Gesù Cristo. Gesù, secondo l'etimologia stessa spesso ricordata da sant'Agostino, è proprio il Salvatore ma non un salvatore qualsiasi bensí un potente e misericordiosissimo Salvatore ${ }^{8}$. Egli ha salvato gratuitamente gli uomini non per la loro giustizia ma per la sua misericordia ${ }^{9}$. Nel contesto della vasta azione salvifica di Dio, che non si limita alla sola dimensione della vita terrena dell'uomo (la connotazione storica) ma trova il suo compimento e la piena realizzazione nella vita eterna e nello stato

${ }^{8}$ Era necessario il Salvatore per la miseria dell'uomo. Cf. Agostino, Espos. sul s. 69, 6 (NBA 26, p. 711); Espos. sul s. 78, 12 (NBA 26, p. 1083). Il Verbo di Dio per mezzo del quale tutte le cose furono create assumendo la carne umana è divenuto il Salvatore. Cf. Idem, Espos. sul s. 49, 1 (NBA 25, p. 1245). Gesù Cristo è Salvatore perchè in lui c'è la salvezza. Cf. Idem, Espos. sul s. 3, 8 (NBA 25, p. 27); Espos. sul s. 49, 31 (NBA 25, p. 1293); Espos. sul s. 71, 14 (NBA 26, p. 811); Espos. sul s. 145, 9 (NBA 28/2, p. 741). E' di Cristo il disegno di accecare i superbi e di salvare gli umili. Cf. Idem, Espos. sul s. 65, 10 (NBA 26, p. 509). In lui viene riconosciuta la salvezza da vecchio Simeone: Espos. sul s. 39, 18 (NBA 25, p. 959). Cf. pure: S. Jaśkiewicz, La misericordia di Dio nelle Confessioni di sant'Agostino, 85 "Studia Ephemeridis Augustinianum", p. 261; M. Pellegrino, "Salus tua ego sum" (Ps 34, 3; Conf I, 5; IX, 1). Il problema della salvezza nelle Confessioni di Sant'Agostino, "Augustinianum" 7 (1967) nr 2, p. 253; H. Rondet, La théologie de S. Augustin prédicateur, "Bulletin de Littérature Ecclésiastique" 72 (1971), p. 247; B. Studer, Dio Salvatore nei Padri della Chiesa, Roma 1986, p. 103-105.

${ }^{9}$ „E tuttavia, per evitare egli stesso di insuperbirsi, e quasi non sembri giustificarsi, continua dicendo: salvami nella tua misericordia, cioè salvami non nella mia giustizia, non per i miei meriti, ma nella tua misericordia; non perché io ne sono degno, ma perché tu sei misericordioso", Agostino, Espos. sul s. 30, II, s. 3, 3 (NBA 25, p. 489). Il Salvatore è fonte della salvezza ma anche della speranza di tutti i poveri ed i bisognosi. Cf. Idem, Espos. sul s. 93, 7 (NBA 27/1, p. 255). 
definitivo di beatitudine (la connotazione escatologica), il vescovo d'Ippona s'impegnò particolarmente a scrutare l'opera storica della redenzione compiuta da Gesù Cristo che permette all'uomo prigioniero la liberazione dalla schiavitù e il ritorno alla comunione con Dio. Perciò, nel contesto della Chiesa pellegrina sulla terra dice: „Dio vide con occhio di misericordia questa pellegrina e, senza che gli uomini lo ricercassero, si mise in loro ricerca. Come li ricercò? Chi mandò nel luogo della nostra prigionia? Mandò il Redentore, secondo la parola dell'Apostolo: Dio inculca il suo amore per noi, poiché Cristo è morto per noi quando eravamo ancora peccatori $(\mathrm{Rm} 5,8)$. A noi prigionieri mandò dunque il Redentore, nella persona del suo Figlio, e (gli) disse: Pòrtati la borsa, mettici dentro il prezzo di quei prigionieri" ${ }^{\prime 0}$.

L'azione redentrice di Dio, che nell'Antico Testamento fu primariamente quella di un favore d'affrancamento sia del singolo che del popolo d'Israele di carattere storico-esistenziale, in Cristo Redentore è giunta alla sua manifestazione piena e definitiva di carattere morale-escatologico. Il titolo di "Redentore" attribuito a Cristo viene spesso scambiato da sant'Agostino con un altro titolo biblico, quello di "Liberatore", in quanto il sottofondo dell'azione redentrice è quello di una dipendenza, di una prigionia ${ }^{11}$. Se nell'essere il Redentore s'intrecciano tutti i ruoli svolti da Cristo a favore degli uomini, tra i quali primariamente si evidenziano quelli di mediatore e di sacerdote, costituendo il punto essenziale del mistero della sua morte cruenta, tanto più si rivela tutta la grandezza dell'amore misericordioso di Dio ${ }^{12}$.

Il papa Giovanni Paolo II ha voluto rendere evidente fin dall'inizio del suo pontificato il nesso esistente tra la vita d'ogni cristiano e Cristo Redentore. Costante è il suo invito a guardare „,verso Cristo, Redentore dell'uomo; verso Cristo, Redentore del mondo. A Lui vogliamo guardare, perché solo in Lui,

${ }^{10}$ Agostino, Espos. sul s. 146, 4 (NBA 28/2, p. 773).

${ }^{11}$ „Se diamo a lui il nome di Redentore, vuol dire che noi eravamo dei prigionieri. Prigionieri in qual posto, per cui egli dovesse venire da noi e liberarci? Dove eravamo relegati? Forse tra i barbari? Peggiori dei barbari sono il diavolo e i suoi angeli, e costoro tenevano prigioniera l'umanità. Per redimerci da questi nemici Cristo ha dato, non oro e argento, ma il suo stesso sangue", Agostino, Espos. sul s. 125, 1 (NBA 28/1, p. 109). Inoltre: „Ma è venuto Lui, e ha chiamato, e ha redento, e ha versato il sangue; ha pagato questo prezzo, ha fatto questo bene, ha patito queste sofferenze", Idem, Espos. sul s 36, s. 2,15 (NBA 25, p. 787). Cf. A. Vassallo, Inquietum cor. Con Agostino alla ricerca di Dio, Palermo 1988, p. 65-72.

${ }^{12}$ „Si le Christ est venu nous montrer l'amour de Dieu, cet enseignement le conduit tout droit à la Pâque où il révélera incomparablement cet amour miséricordieux. C'est bien la principale raison de sa venue que de nous montrer cette tendresse, cet amour prévenant: Dieu livrant son Fils unique, qui ne craint pas de mourir pour des pécheurs, appellant la rèponse de notre propre amour", J.-P. Jossua, Le salut, Incarnation ou Mystère Pascal. (Chez les Pères de l'Église de saint Irénée à saint Léon le Grand), Paris 1968, p. 200. Cf. S. Jaśkiewicz, La misericordia, cit., p. 262. 
Figlio di Dio, c'è salvezza, rinnovando l'affermazione di Pietro: «Signore, a chi andremo? Tu hai parole di vita eterna» (Gv 6,68; cf. At 4,8-12)" (RH 7). Il Redentore dell'uomo è veramente nobile e grande. Il Pontefice usa l'espressione „Redentore dell'uomo" perchè è proprio Gesù Cristo non solo ad assicurare all'uomo il suo destino eterno, ma anche dà la forza per raggiungerlo. Cristo è il Redentore non solo dell'anima dell'uomo ma dell'uomo intero, e tanto più non solo per la sua salvezza finale, ma anche per questo tempo di esistenza che percorre nelle più varie condizioni terrene.

\section{L'atto redentore di Cristo}

La Redenzione operata da Cristo, vista da sant'Agostino come un'eminente opera di riconciliazione dell'uomo peccatore con Dio, risultante insieme dell'amore, della giustizia e della misericordia divine, si è compiuta attraverso la sua morte. Il vescovo d'Ippona, esaminando il significato e le finalità della morte cruenta di Cristo, riunisce gli schemi e le idee diverse facendo leva soprattutto sull'idea di sacrificio. La passione e la morte volontaria sono il grande sacrificio che Cristo Sacerdote e Mediatore, secondo gli schemi della mediazione ascendente, ha offerto per la salvezza dell'umanità che dopo il peccato di Adamo è diventata una grande "massa di peccatori". Secondo le coordinate della sua visione soteriologica, sant'Agostino assegna un ruolo primario al valore «espiatorio» della sua morte. L'unico sacrificio di Cristo Sacerdote nella sua natura umana fu già prefigurato nell'Antico Testamento dai sacrifici (lo spargimento del sangue delle vittime) che i sacerdoti offrivano a Yahve per renderlo propizio al Popolo eletto, e in particolare da quello di Melchisedec. Così, viene spiegato: „Tu sarai propizio alle nostre empietà, non si possono dire se non di un sacerdote che offre qualcosa con cui l'empietà sia espiata e perdonata. Infatti si dice che si propizia una empietà quando il Signore diviene propizio verso tale empietà. E come si fa a rendere Dio propizio verso le empietà, ottenere cioè che le perdoni e conceda venia? Per ottenere perdono dal Signore si compie la propiziazione con qualche sacrificio. Esiste dunque, mandato dal Signore Dio, un misterioso nostro sacerdote"13.

${ }^{13}$ Agostino, Espos. sul s. 64, 6 (NBA 26, p. 467). Cf. Idem, Espos. sul s. 36, s. 2, 20 (NBA 25, p. 799). A sant'Agostino s'aplica bene ciò che N. Cipriani afferma in modo assai generale riguardo ai diversi Padri: „Un'idea assai ricorrente è quella della sostituzione, dello scambio o della morte vicaria: Gesù Cristo è morto per noi, sostituendosi a noi, che meritavamo la morte a causa dei peccati”, Idem, Gesù il Figlio, cit., p.15. Cf. É. Bailleux, La sotériologie, cit., p. 161; A.F. Krüeger, Synthesis of sacrifice according to saint Augustine. A Study of the sacramentality of sacrifice, (Diss. St. Mary of the Lake, Illinois), Mundelein 1950, p. 81; A. Sanguinetti Montero, 


\section{The Person and the Challenges \\ 214 Volume 2 (2012) Number 1}

Dio, come risulta dalla storia del Popolo eletto, non rimane indifferente verso chi trasgredisce la sua legge ma dall'altro lato non serba la sua ira e il suo sdegno in eterno, dimenticando la sua misericordia e la sua benevolenza. Egli non impedisce che il sacrificio e la preghiera abbiano un loro valore e ottengano il suo favore. Dal tempo di Aronne, per rendere Dio propizio di fronte ad un'empietà, occorreva un sacrificio offerto da un suo discendente appartenente alla tribù di Levi. Nel caso dell'offesa di Dio, risultante dal primo peccato, non era sufficiente nè un'offerta nè un sacerdote consueti per riacquistare l'amicizia perduta, e perciò Cristo in sostituzione ha offerto in oblazione sacrificale se stesso. Cosí come nell'Antico Testamento il sommo sacerdote entrava nella festa dell'Espiazione nel santuario per aspergere col sangue degli animali il propiziatorio, cosí il Cristo col proprio sangue sparso nel santuario della Nuova Alleanza ha ottenuto i benefici che vanno ben oltre la semplice purificazione, costituendo l'unione definitiva tra Dio e gli uomini ${ }^{14}$. L'unico sacrificio di Cristo, sotto l'aspetto soggettivo vero e libero e quello oggettivo completo e di efficacia assoluta, cioè perfetto, è composto da quattro elementi essenziali: il sacerdote, la vittima, il sacrificatore, il sacrificio ${ }^{15}$.

Gratuidad y respuesta del hombre a Dios. Estudio en las "Enarrationes in Psalmos" de san Agustín, Montevideo 1983, p. 148-150; B. Studer, Espiazione, in: Dizionario Patristico e di Antichità Cristiane, ed. A. Di Berardino, vol. I, Casale Monferrato 1983, p. 1234. Il tema del sacrificio è in sant'Agostino uno dei temi centrali e ne parla in tutte le sue opere principali. Egli fu convinto che fin dall'antichità il culto verso Dio si sia espresso attraverso i sacrifici e perciò tratta del sacrificio dell'altare, del sacrificio universale della redempta civitas, e dell'unico sacrificio della croce. Cf. E. Aliaga Girbés, El sacrificio sacerdotal de Jesucristo en las "Enarrationes in Psalmos" de San Agustín, "Anales valentines" 1 (1975), p. 22-25; B. Mondin, L'uomo, cit., p. 262; H.E.W. Turner, Jésus le Sauveur. Essai sur la doctrine patristique de la Rédemption, Paris 1965, p. 114.

${ }^{14}$ „Prefigurando ciò, in quel primo popolo e in quel primo tempio, il solo sacredote entrava nel santo dei santi, mentre tutto il popolo restava fuori. E colui che, solo, era entrato al di là del velo, offriva il sacrificio per il popolo che stava fuori (cf. Eb 6,19-20)", Agostino, Espos. sul s. 64, 6 (NBA 25, p. 467). In modo simile: „Eppure è certo che abbiamo un sacerdote. Lo abbiamo nel cielo, dove interpella il Padre a nostro favore (cf. Eb 9,12). Egli entrò nel santo dei santi, al di là del velo, dove il pontefice-simbolo, non entrava se non una volta all'anno: come, del resto, anche il Signore nell'intero arco della sua vita, egli fu immolato soltanto una volta. Sacerdote e insieme vittima, egli offrí se stesso ed entrò una volta nel santo dei santi e da allora egli piú non muore né la morte ha alcun potere su di lui", Idem, Espos. sul s. 130, 4 (NBA 28/1, p. 243-245). Cf. G. Favara, Chiesa e preghiera in sant'Agostino, "Palestra del Clero" 36 (1957), p. 554-556.

${ }^{15}$ „Tu sacerdote, tu vittima, tu sacrificatore, tu sacrificio. Egli è il sacerdote che ora è entrato al di là del velo e, solo fra tutti coloro che hanno portato la carne, intercede per noi (cf. Eb 6,1920)", Agostino, Espos. sul s. 64, 6 (NBA 26, p. 467). Cf. Idem, Espos. sul s. 2, 7 (NBA 25, p. 15). Parlando dei diversi componenti dell'unico sacrificio vicario di Cristo non si può prescindere dal suo carattere interiore che mette in giusta luce tutti gli elementi (libertà, ubbedienza, umiltà, ecc.) anche quelli esteriori. Cf. A.F. Krüeger, Synthesis of sacrifice, cit., p. 86-88; B. Mondin, L'uomo, cit., p. 262-263; A. Pollastri, Sacrificio, in: Dizionario Patristico, cit., p. 3060-3062; E. Portalié, Augustin (Saint), in: Dictionnaire de Théologie Catholique, ed. A. Vacant, E. Mangenot, Paris 
Esso è stato offerto da Cristo a Dio, il quale ha accettato questa sostituzione al posto degli uomini. Spiega sant'Agostino: „Egli prese da noi la vittima da offrire al Signore, prese ciò che noi abbiamo chiamato le sante primizie della carne dal seno della Vergine. Questo olocausto che egli offrí a Dio, quando stese le sue mani sulla croce per dire: Si levi la mia preghiera come incenso al tuo cospetto; il levarsi delle mie mani sia quale sacificio vespertino (S1 140, 2). Come voi sapete, era sera quando il Signore fu sospeso alla croce (cf. Mt 27, 46); e da quel momento le nostre empietà sono state espiate" ${ }^{" 16}$.

Il sacrificio della croce assume nella riflessione agostiniana svariate valenze. Esso, in quanto realizza lo scopo di tutti i sacrifici, cioè il ritorno dell'uomo a Dio e non si limita alla dimensione singolare ed esemplare, è un vero sacramento ${ }^{17}$. Tale sacrificio trova il suo prolungamento storico nel sacrificio della Chiesa, cioè nel mistero eucaristico.

Un'altra dimensione scritturistica della morte cruenta di Cristo, presente fin dai primi momenti dell'attività letteraria cristiana, è quella di riscatto, ossia del prezzo del sangue versato da Cristo. In quest'idea risuonano le categorie tipicamente commerciali o giuridiche, in quanto la morte di Cristo viene

1980, p. 2369-2370; E. Scano, Il Cristocentrismo e $i$ suoi fondamenti dogmatici in s. Agostino, Torino 1951, p. 119-120; H.E.W. Turner, Jésus le Sauveur, cit., p. 114-115.

${ }^{16}$ Agostino, Espos. sul s. 64, 6 (NBA 26, p. 467). Cristo era venuto nell'umiltà a soffrire ciò che avrebbero da soffrire i peccatori. Cf. Idem, Espos. sul s. 93, 8 (NBA 27/1, p. 257). Egli è diventato l'uomo, cioè: „Egli prese le primizie della carne dal seno della Vergine (una volta assunte queste primizie il resto ha da venire di conseguenza) per offrire un olocausto completo", Idem, Espos. sul s. 64, 5 (NBA 26, p. 465). Il sacrificio di espiazione offerto da Cristo sacerdote rivela la sua fondamentale dimensione sostitutiva o vicaria in considerazione dell'incapacità dell'uomo. Partendo dalle conseguenze del peccato originale A.F. Krüeger lo spiega cosí: „Sin is an offense against God's honor. As a result of sin man must pay a two-fold dept; he must pay back the honor taken from God, and the penalty for that stolen honor. In other words he must, by Divine decree propitiate God through sacrifice. Man himself is unfit for such a task. Another must do it, his representative, his vicar. That Christ was man's representative must be evident. It remains to consider how He fulfilled His mission", Synthesis of sacrifice, cit., p. 81. Cf. Ch. Boyer, Le triple amour du Christ pour les hommes dans les écrits de Saint Augustin, in: Cor Jesu, Commentationes in litteras Encyclicas Pii PP. XII "Haurietis Aquas", ed. A. Bea - H. Rahner, vol. I, Roma 1959, p. 590; H. Rondet, La théologie, cit., p. 247; B. Sesboüé, Storia dei Dogmi, vol. I, ed. B. Sesboüé (et al.), Casale Monferrato 1997, p. 421.

${ }^{17}$ Cosí come la morte e la risurrezione di Cristo sono i sacramenti della morte e della risurrezione dell'uomo, cosí anche „,...) c'est-à-dire que le sacrifice du Christ n'est pas seulement un modèle à suivre, un exemplaire de nos sacrifices personnels, mais qu'il les signifie et les cause tout ensemble; la juxtaposition des deux mots: sacramentum-exemplum montre assez qu'il n'y s'agit pas seulement d'une signification vide et inefficace, mais bien d'une réelle causalité", J. Lécuyer, Le sacrifice selon saint Augustin, in: Augustinus Magister. Congrès International Augustinien, (Paris, 21-24 Sept. 1954), vol. II, Paris 1954, p. 909. Cf. B. Sesboüé, Gesù Cristo l'unico mediatore, Cinisello Balsamo 1991, p. 309. 
interpretata come il riscatto e il sangue di Cristo come suo prezzo. Su questo filone di pensiero il vescovo d'Ippona inserisce le sue frequenti spiegazioni sui dettagli di «comercium emptionis» ${ }^{18}$. Per quanto riguarda l'oggetto del riscatto oppure ciò da che cosa si riscatta accanto alla schiavitù del peccato si parla del riscatto dalla giusta ira di $\mathrm{Dio}^{19}$. In tal modo, attraverso la morte cruenta di Cristo, l'ira divina viene placata. Il vescovo d'Ippona, da una parte, per il suo particolare impegno nelle questioni riguardanti il peccato di Adamo e, dall'altra, per il suo legame con le tradizioni popolari, inserì ben presto, anche se non in maniera così pittoresca come san Ireneo o Origene, il riferimento al demonio, che secondo le tradizioni più antiche teneva l'uomo peccatore sotto il suo dominio. La morte cruenta di Cristo, il suo sangue innocente ha contribuito ai numerosi benefici. Il vescovo d'Ippona sottolinea la sconfitta definitiva del demonio: „Ecco però che, crocifisso Cristo, l'umanità è redenta: sicché il furore che animava il diavolo si cambiò in danno per lui e in vantaggio per noi. Col suo accanimento infatti egli perse l'uomo che teneva sotto di sé e che venne liberato mediante il sangue del Signore che egli fece versare spinto dalla sua propria rabbia. Se avesse conosciuto il gravissimo danno che gliene derivava, non avrebbe certo fatto versare il prezzo con cui sarebbe stato redento il genere umano" 20 .

${ }^{18}$ Questi dettagli il più delle volte sembrano esser incompleti o parziali come ad es. nel commento al salmo 147,14 dove nel contesto dell'idea centrale del possedimento del Signore si tratta del compratore, del prezzo e dell'oggetto acquistato ma viene a mancare il proprietaio: „Osserva lo scambio che ha portato al nostro riscatto. Cristo pende sulla croce: guarda qual prezzo sborsa per il riscatto e cosí vedrai cos'abbia riscattato. Sta per acquistare qualcosa: di che si tratti, non lo sai ancora. Guarda, guarda al prezzo e vedrai di che si tratta. Versò il sangue e col suo sangue operò il riscatto: riscattò al prezzo del sangue dell'Agnello immacolato, riscattò al prezzo del sangue del Figlio unigenito di Dio. (...) Il compratore è Cristo; prezzo è il sangue, acquisto l'universo", Agostino, Espos. sul s. 147, 16 (NBA 28/2, p. 833). In questo contesto trovano il loro fondamento i titoli "Christus manumissor" e "Christus mercator", tratti da sant'Agostino dal campo delle nozioni giuridico-mercantili. Cf. S. Poque, Christus Mercator. Notes et mélanges. Notes augustiniennes, "Recherches de Science Religieuse" 48 (1960), p. 564-577; A. Viciano, Titoli soterici di Cristo nei "Sermones" di sant'Agostino, in: Congresso Internazionale su s. Agostino nel XVI centenario della conversione, (Roma 15-20 sett. 1986), vol. II, Roma 1987, p. 334.

${ }^{19}$ Cristo che si è fatto fratello d'ogni uomo tutti ha riscattato dalla giusta ira divina: „Alcuni si fidano dei loro amici; altri si fidano della loro forza, altri ancora delle ricchezze. Questa è la presunzione del genere umano che non si fida di Dio. Ha parlato della forza, ha parlato delle ricchezze, ora parla degli amici: Non riscatta il fratello, riscatterà l'uomo? Speri che l'uomo ti riscatti dall'ira che verrà? Se il fratello non ti riscatta, ti riscatterà l'uomo? Chi è il fratello che, se non riscatterà, nessun uomo sarà riscattato? É colui che dopo la resurrezione dice: $V a$ ', di' ai miei fratelli (Mt 28, 10)», Agostino, Espos. sul s. 48, s. 1, 8 (NBA 25, p. 1205-1207). Cf. J.N.D. Kelly, Il pensiero delle origini, Bologna 1972, p. 482; H. Rondet, La théologie, cit., p. 247.

\footnotetext{
${ }^{20}$ Agostino, Espos. sul s. 130, 7 (NBA 28/1, p. 251). Cf. H. Rondet, La théologie, cit., p. 246.
} 


\section{Il demonio viene vinto da Cristo non attraverso la sua potenza ma mediante la} sua innocenza e giustizia ${ }^{21}$. Nella vittoria di Cristo sul demonio c'è la vittoria di ogni uomo ${ }^{22}$. Un ruolo importante, nel fondare il valore permanente del sacrificio

${ }^{21}$ „Patisco, dice, incolpevole per i colpevoli, per fare degni della mia vita coloro per i quali senza colpa alcuna subisco la morte che ad essi compete", Agostino, Espos. sul s. 50, 9 (NBA 25, p. 1307). Cf. Idem, Enarr. in Ps. 140, 3 (CCL 40, p. 2027). Nell'Espos. sul s. 106, 3 (NBA 27/2, p. 545), sant'Agostino dimostra un suggestivo parallelismo tra gli episodi della storia della liberazione degli Ebrei dall'Egitto ed i misteri dell'opera salvifica di Cristo. Il vescovo d'Ippona ancorato ai canoni tradizionali non rinuncia del tutto, nel trattare della vittoria di Cristo, al ricorso alle forme e alle immagini secondarie di esposizione conformi alla mentalità popolare. Ne sono state fondate in particolare da san Ireneo e Origene almeno due, quella dei «diritti del demonio» o dell' «abuso del potere del demonio». Secondo la prima teoria Cristo, per riscattare tutti gli uomini dalla schiavitù del demonio, pagò a lui il prezzo del suo sangue. La seconda si basa prevalentemente sull'idea della giustizia di Cristo. L'essere giustificati nel sangue di Cristo $(\mathrm{Rm} 5,9)$ significa essere liberati a motivo della giustizia di Cristo innocentemente ucciso dal demonio. Il demonio si è ingannato perché voleva provare che anche Cristo era come tutti gli altri. J. Rivière analizzando tutte e due le teorie nel contesto dell'elaborazione agostiniana sottolinea l'importanza del suo contributo: „L'originalité de saint Augustin dans l'histoire de la théologie rédemptrice n'est donc pas d'avoir échappe - et encore par un simple hasard - à la grossière conception du marché frauduleux, mais d'avoir au moins tacitement évincé la théorie pseudo-juridique de la rançon, qui disparait dès lors sans retour, pour lui substituer la doctrine de l'abus de pouvoir, dont il existait tout au plus avant lui des éléments. C'est, en effet, celle-ci qui devient désormais courante dans la suite, jusqu'au moment où elle fut à son tour discréditée par les critiques de saint Anselme et d'Abélard", Idem, Le dogme de la rédemption chez saint Augustin, Paris 1933, p. 253. Nello studio dei testi agostiniani sulla redenzione e in particolare di quelli numerosi sul riscatto nel contesto del dominio del demonio, come sottolinea H. Rondet (La théologie, cit., p. 246) si deve mantenere un sano equilibrio per non cadere nel vicolo cieco come è capitato secondo lui al sopra citato J. Rivière, il quale s'è messo esclusivamente ad esaminare il ruolo del demonio (la teoria dei diritti del demonio) nella concezione agostiniana della redenzione. Cf. M. Bordoni, Gesù di Nazaret, Signore e Cristo, vol. III. Il Cristo annunciatore della Chiesa, Roma 1982, p. 289. Intanto giova notare ciò che sul prezzo del sangue pagato da Cristo assolutamente libero e il suo destinatario sottolinea É. Bailleux: „Lorsque le Médiateur de la vie, cédant à la violance exercée contre lui par le médiateur de la mort, consent librement à mourir, il s'immole lui-même à Dieu pour notre salut. Le sang précieux du Sauveur n'est donc nullement offert au démon: un tel sacrifice serait sacriliège. Pourtant, sans en avoir le droit, le médiateur de la mort a versé le sang du Christ. Or, comme le Christ l'a laissé faire, parce que cet abus de pouvoir servait son dessein rédempteur, on peut dire avec saint Augustin que le démon a reçu ce sang pour prix de notre liberation; mais personne ne lui a donné cette rançon qu'il a du reste touchée pour sa perte", La sotériologie, cit., p.168. Cf. B. Altaner, Patrologia, Torino 1952, p. 324; J.-P. Jossua, Le salut, cit., p. 174-187; A.F. Krüeger, Synthesis of sacrifice, cit., p. 93-95; G. Remy, Le Christ mediateur, cit., p. 472-476; B. Sesboüé, Gesù Cristo l'unico mediatore, cit., p. 174-176; D. Spada, La misericordia nel pensiero di san Tommaso d'Aquino, Dives in misericordia. Commento all'enciclica di Giovanni Paolo II, Roma 1981, p. 214.

${ }^{22}$ „Cristo fu certamente tentato dal diavolo, ma in Cristo eri tentato tu. Tua infatti era la carne che Cristo aveva presa perché tu avessi da lui la salvezza. Egli aveva preso per sé la morte, che era tua, per donare a te la vita; da te egli aveva preso su di sé le umiliazioni perché tu avessi da lui la gloria. Cosí, egli prese da te e fece sua tentazione, affinché per suo dono tu ne riportassi vittoria. (...) Egli avrebbe potuto tener lontano da sé il diavolo; ma, se non si fosse lasciato tentare, non ti avrebbe insegnato a vincere quando tu sei tentato", Agostino, Espos. sul s. 60, 3 (NBA 26, 


\section{The Person and the Challenges \\ 218 Volume 2 (2012) Number 1}

di Cristo nella soteriologia dell'Ipponate, compie la dottrina agostiniana della «rappresentanza» nell'ambito della tematica ecclesiologica, che concepisce tutto il movimento sacrifiale cristiano. Nel sacrificio di Cristo, in quanto sacrificio del Capo, sono stati rappresentati o inclusi (dimensione vicaria) tutti i cristiani in quanto suo corpo, sua Chiesa ${ }^{23}$. Il sacrificio di Cristo Capo si attualizza nella sua Chiesa attraverso l'Eucaristia facendone partecipi tutti i cristiani, i quali, avendo la vita nuova, cantano il cantico nuovo ${ }^{24}$. L'opera redentrice di Cristo ontologicamente fondata sull'incarnazione, rivela anche la sua dimensione morale di conversione dei cuori umani attraverso la scuola della sua profonda umiltà che, in senso stretto, non è più l'opera di uomo-Dio ma di Dio-uomo. In tale senso: „Che cosa altro infatti ha insegnato umiliandosi, fattosi obbediente fino alla morte, e alla morte di croce (Fil 2, 8)? Che cosa altro ha insegnato pagando ciò che non doveva, onde liberare noi dal nostro debito? Che cosa altro ha insegnato facendosi battezzare pur non avendo commesso peccato, lasciandosi crocifiggere pur non avendo colpa alcuna? Giustamente dice: Io sono la via, la verità, la vita (Gv 14, 6). In questa umiltà, dunque, ci si avvicina a Dio, perché vicino è il Signore a coloro che hanno il cuore contrito (cf. Sl 33, 19)"25. L'umiltà

p. 327). Cf. Idem, Espos. sul s. 90, s. 2,7 (NBA 27/1, p. 175). Il tema delle tentazioni riveste in sant'Agostino maggiore attualità nella polemica antipelagiana. Cf. T.J. van Bavel, Recherches sur la christologie de saint Augustin, Fribourg 1954, p. 137; M. Vincent, Saint Augustine, maître de prière d'après les Enarrationes in Psalmos, Paris 1990, p. 285-290.

${ }^{23}$ „Perché Gesú Cristo, capo e corpo, è un uomo solo: è salvatore del corpo e membra del corpo, due in una sola carne (cf. Gn 2,24; Ef 5,31), in un'unica voce e in un'unica sofferenza; e, quando l'iniquità sarà scomparsa, in una sola pace. Perciò le sofferenze di Cristo non sono esclusivamente nel Cristo, o meglio, le sofferenze di Cristo non possono essere se non nel Cristo. Se intendi Cristo come capo e corpo, non vi sono sofferenze al di fuori di Cristo; se, invece, intendi Cristo soltanto come capo, le sofferenze di Cristo non le troviamo esclusivamente nel Cristo", Agostino, Espos. sul s. 61, 4 (NBA 26, p. 343-345). Cf. M. Bordoni, Gesù di Nazaret, cit., p. 308-309.

${ }^{24}$ „Sono rimasti nell'ombra, non possano sopportare il sole della gloria; noi invece siamo ormai nella luce, abbiamo il corpo di Cristo, possediamo il sangue di Cristo", Agostino, Espos. sul s. 39, 13 (NBA 25, p. 953). Sulla croce viene preannunciato il sacrificio dell'altare perchè Cristo venga immolato ogni giorno per gli uomini. Cf. Idem, Espos. sul s. 33, I, 6 (NBA 25, p. 623); Espos. sul s. 75, 15 (NBA 26, p. 959). Cf. G. Remy, Le Christ mediateur, cit., p. 481-482.

${ }^{25}$ „Che cosa altro infatti ha insegnato umiliandosi, fattosi obbediente fino alla morte, e alla morte di Croce (Fil 2,8)? Che cosa altro ha insegnato pagando ciò che non doveva, onde liberare noi dal nostro debito? Che cosa altro ha insegnato facendosi battezzare pur non avendo commesso peccato, lasciandosi crocifiggere pur non avendo colpa alcuna? Che cosa altro ha insegnato, se non questa umiltà? Giustamente dice: Io sono la via, la verità, la vita (Gv 14,6). In questa umiltà, dunque, ci si avvicina a Dio, perché vicino è il Signore a coloro che hanno il cuore contrito (cf. Sl 33,19)", Agostino, Espos. sul s. 31, II, 18 (NBA 25, p. 541). L'umiltà di Cristo è stata ben raffigurata dal suo reclinare il capo. E' una grande lezione d'umiltà che Cristo, essendo Dio, si è fatto uomo. Cf. Idem, Espos. sul s. 93, 15 (NBA 27/1, p. 273). Come sottolinea E. Portalié: „De tous les Pères, sans contredit, nul n'a développé avec autant d'insistance qu'Augustin ce côté moral de 
di Cristo, in quanto polo opposto della superbia del primo uomo, non è un atto singolare ma si estende a tutta la sua esistenza terrena, trovando il suo punto culminante nella sua morte in croce.

Gesù Cristo per redimerci ha offerto se stesso sulla croce. Ecco, allora con quali parole Giovanni Paolo II ha descritto da parte sua la dimensione divina del mistero della redenzione di Cristo: "Proprio Lui, solo Lui ha soddisfatto all'eterno amore del Padre, a quella paternità che sin dal principio si è espressa nella creazione del mondo, nella donazione all'uomo di tutta la ricchezza del creato, nel farlo «poco meno degli angeli» (Sal 8,6), in quanto creato «ad immagine ed a somiglianza di Dio» (cf. Gn 1,26); e, egualmente, ha soddisfatto a quella paternità di Dio e a quell'amore, in un certo modo respinto dall'uomo con la rottura della prima Alleanza (cf. Gn 3,6-13) e di quelle posteriori che Dio «molte volte ha offerto agli uomini» (cf. IV Prece eucaristica)" (RH 9). Giovanni Paolo II ribadendo quanto affermato già dal vescovo d'Ippona, ha così voluto sottolineare che l'atto redentore di Cristo „è, nella sua più profonda radice, la pienezza della giustizia in un Cuore umano: nel Cuore del Figlio primogenito, perché essa possa diventare giustizia dei cuori di molti uomini, i quali proprio nel Figlio primogenito sono stati, fin dall'eternità, predestinati a divenire figli di Dio (cf. Rm 8,29ss.; Ef 1,8) e chiamati alla grazia, chiamati all'amore" (RH 9). L'atto redentore di Cristo, nella prospettiva di Giovanni Paolo II è sempre la rivelazione dell'amore e della misericordia di Dio.

\section{La liberazione operata da Cristo}

Il punto di partenza per trattare la redenzione, ossia la liberazione operata da Cristo, consiste necessariamente nell'affermazione del peccato di Adamo e delle sue conseguenze riguardanti tutti gli uomini. Al vescovo d'Ippona, zelante difensore del peccato originale, non era nuova la verità, oramai ben formulata dai suoi predecessori, che a causa del peccato di Adamo, per giusto giudizio di Dio, tutto il genere umano è caduto nel potere del demonio. Il demonio, a cui un certo potere è stato consentito da Dio, teneva nelle sue mani i peccati degli uomini e, per mezzo di essi, il regno della morte. Perciò il beneficio della vittoria di Cristo

l'incarnation. C'est même là un cachet tout personnel de sa doctrine: sa thèse sur l'humilité de Dieu dans l'incarnation est une de ses plus profondes conceptions", Idem, Augustin (Saint), cit., p. 2372. Cf. É. Bailleux, La sotériologie, cit., p. 171; J.-P. Jossua, Le salut, cit., p. 196-201; A.F. Krüeger, Synthesis of sacrifice, cit., p. 84-86.96-97; B. Mondin, Dio: Chi è? Elementi di teologia filosofica, Milano 1990, p. 325-326; T. van Bavel, L'humanité du Christ comme lac parvulorm et comme via dans la spiritualité de saint Augustin, "Augustinus" 7 (1957), p. 253. 
sul peccato, avvenuta attraverso il sacrificio della croce, ha a che fare con la triplice liberazione degli uomini: dal peccato, dalla morte e dal demonio. Tutti e tre sono vere e proprie schiavitù dell'uomo, come sottolinea il vescovo d'Ippona esaminando i dati della Scrittura. Nel contesto di essi l'opera di Cristo assume il significato di una vera vittoria che pone il termine definitivo alle schiavitù antiche dell'uomo, invece a Cristo conferisce il titolo di vero Re e Sacerdote ${ }^{26}$. Se il primo uomo è stato giustamente la causa della prigionia di tutti gli uomini per la sua disobbedienza, il secondo Uomo, totalmete libero, attraverso il suo sacrificio e totale obbedienza al Padre, ha aperto le porte della prigione e ha fatto uscirne quanti lì giacevano. All'idea della ricapitolazione viene associata dal vescovo d'Ippona quella della sostituzione, per indicare che Cristo, condividendo la natura umana, si è messo al posto degli uomini per subire la punizione a loro dovuta. Cristo muore per fare la volontà del Padre: „Ma affinché tutti sappiano - diceva ancora - che io faccio la volontà del Padre mio, levatevi, andiamocene da qui (Gv 14, 31). E voleva dire: Se muoio, è per fare la volontà del Padre mio; non che io sia reo di morte. Non ho fatto nulla per cui debba morire, ma faccio in modo di morire, affinché per la morte dell'innocente siano liberati coloro che meritavano di morire" 27 .

Il peccato di Adamo ha reso peccatori tutti gli uomini. Cristo diede se stesso alla morte sulla croce al posto degli uomini, liberandoli cosí dai loro peccati. La

${ }^{26}$ „Quando combatté per noi sembrò quasi che fosse vinto, in realtà però fu vincitore. Fu sí crocifisso, però dalla croce in cui era confitto uccise il diavolo, e per questo è nostro re. Perché poi è sacerdote? Per aver offerto se stesso per noi", Agostino, Espos. sul s. 149, 6 (NBA 28/2, p. 903). La dottrina del Cristo vincitore è ben nota nell'antichità cristiana rivelando spesso il suo legame con le religioni pagane, con la mitologia o la filosofia come ad. es. in Lattanzio o Arnobio. Nel terzo secolo, in cui i veri promotori sono Tertulliano e Cipriano si presenta la liberazione come una grande vittoria di Cristo anche se il vocabolario è ancora molto ridotto. Un altro africano, quasi un secolo dopo Mario Vittorino, non solo definirà il Verbo di Dio liberator ma anche l'azione salvifica di Cristo chiamerà liberatio. Cf. M. Bordoni, Gesù di Nazaret, cit., p. 289-292; B. Sesboüé, Storia dei dogmi, vol. I, cit., p. 412.415; A. Viciano, Titoli soterici, cit., p. 323-335.

${ }^{27}$ Agostino, Espos. sul s. 142, 8 (NBA 28/2, p. 635). Grazie al sacrificio di Cristo, alla sua morte, i peccati umani sono stati perdonati: „Quanto a noi, perdonati i nostri peccati e le nostre empietà grazie a quel sacrificio vespertino, passiamo al Signore e il velo c'è tolto, tanto è vero che, quando il Signore fu crocifisso, il velo del tempio si squarciò (cf. Mt 27, 51)", Idem, Espos. sul s. 64, 6 (NBA 26, p. 469). Tra due uomini: „Il primo uomo ci ha fatti prigionieri; il secondo uomo ci ha liberati dalla prigionia", Idem, Espos. sul s. 70, s. 1, 2 (NBA 26, p. 725). A.F. Krüeger analizzando l'esegesi agostiniana della II Co 5,21 dove san Paolo dice che Dio fece Cristo peccato per gli uomini indica tre interpretazioni agostiniane della parola peccatum ossia della vittoria definitiva operata da Cristo. Il peccato significherebbe: sacrificium pro peccato - il sacrificio sostitutivo di Cristo per il peccato dell'uomo, peccatum - direttamente il peccato nel contesto della libertà dalla schiavitù operata da Cristo, medium separans - un ostacolo, un mezzo di separazione tra Dio e gli uomini distrutto dal Cristo disteso sulla croce. Cf. Idem, Synthesis of sacrifice, cit., p. 89-92. 
liberazione di ogni singolo uomo dalla colpa originale è avvenuta non secondo le semplici norme della legge, nè tanto più a motivo dei meriti umani. Essa è una grazia misericordiosa di Cristo, perché è data gratuitamente da colui che non aveva bisogno di essere liberato. Non c'è alcun dubbio „se il Signore operasse fedelmente secondo le norme della legge stabilita, il peccatore dovrebbe esser condannato. Se agisse secondo questa norma, chi libererebbe? Ha trovato tutti peccatori; senza peccato è venuto solo Colui che ci ha trovati peccatori. Cosí dice l'Apostolo: perché tutti hanno peccato e hanno bisogno della gloria di Dio (Rom $3,23)$. Che vuol dire: hanno bisogno della gloria di Dio? Che Egli ti liberi, non tu da te stesso; poiché da te non ti puoi liberare, hai bisogno del liberatore"28.

Il Figlio di Dio fatto uomo attraverso la sua morte innocente ha pagato i debiti che non doveva pagare, liberando in tal modo tutti i debitori. Egli, inserendosi mediante la sua incarnazione nel flusso incessante della vita umana, dovette far fronte anche all'altro fondamentale evento umano che è la morte. Se da un lato la morte di Cristo era in tutto reale ed autentica a motivo della vera carne umana assunta da lui, dall'altro da essa fin dall'inizio spuntava il raggio della vittoria definitiva di Cristo, in quanto Egli, rimanendo non macchiato dal peccato, l'ha subìta liberamente e volontariamente e cosí ha rimediato alla morte dell'uomo. Nel contesto del mistero della morte spiega il vescovo d'Ippona: „Perché non Lui ha portato la morte, ma l'ha trovata trasmessa qui dalla maledizione del primo uomo (cf. Ga 3,10); ed accettando la nostra stessa morte, ha inchiodata al legno quella morte che era venuta per mezzo del peccato. (...) Poiché quella morte non era apparente, ma gli derivava dal propagarsi della maledizione della sua stirpe, allorché Dio aveva detto: Morirete di morte (Gn 2, 17), cosí Egli è giunto pienamente alla vera morte, perché noi pervenissimo alla vera vita (...)”29.

${ }^{28}$ Agostino, Espos. sul s. 30, II, d. 1, 6 (NBA 25, p. 449). Il braccio del Signore che è Cristo ha portato la liberazione gratuita. Cf. Idem, Espos. sul s. 70, d. 2, 5 (NBA 26, p. 771). Cristo non è venuto per trattare dei meriti degli uomini ma per rimettere i loro peccati: „Non è venuto per indagare sui nostri meriti, ma per rimettere i nostri peccati. Non eri e sei stato creato; che cosa hai dato a Dio? Eri malvagio, e sei stato liberato; che cosa hai dato a Dio? Che cosa non hai ricevuto gratuitamente da lui? Giustamente si chiama grazia, perché è data gratuitamente. Si esige dunque da te che anche tu lo adori gratuitamente, non perché elargisce doni temporali, ma perché dona quelli eterni", Idem, Espos. sul s. 43, 15 (NBA 25, p. 1061). Egli ha rimesso i debiti dei peccatori e ha pagato ciò che non doveva pagare. Cf. Idem, Espos. sul s. 44, 7 (NBA 25, p. 1087).

${ }^{29}$ Agostino, Espos. sul s. 37, 26 (NBA 25, p. 877-879). Cf. Idem, Espos. sul s. 109, 20 (NBA 27/2, p. 993). Il ragionamento agostiniano, come sottolinea H.E.W. Turner: „se fonde sur deux postulats: le premier, que le Christ était personnellement innocent de tout péché, deuxièmement, que la mort est la peine du péché. La mort n'a donc pas de prise sur le Christ, et si le Christ meurt, il faut bien que ce soit au bénéfice des autres", Jésus le Sauveur, cit., p. 115. Cf. J.-P. Jossua, Le salut, cit., p. 192-195. 
Il primo peccato ha causato la duplice morte di ogni uomo: dell'anima e quella del corpo, l'empietà della prima e la corruttibilità del secondo. Attraverso la morte di Cristo viene aperto all'uomo il passaggio dal regno della morte verso una vita nuova in Cristo. Nella carne umana, attraverso il sacrum commercium Cristo non solo cancella il peccato, vince la morte, ma la sua vittoria si stende anche sul demonio. Se nell'Eden il demonio aveva vinto il primo uomo stendendo su di lui il suo potere a motivo del peccato, il Cristo, nuovo Adamo, attraverso la sua morte innocente, distrugge il peccato e nello stesso tempo sconfigge definitamente il demonio. Inoltre il Dottore della grazia annota: „E’ a proposito di questa grazia di Dio che il profeta dice, rivolto al Signore Gesú Cristo: Hai allontanato la schiavitú da Giacobbe. Ponete mente a questa schiavitú di Giacobbe. Badate che «allontanare da noi la schiavitúı non consiste nell'averci liberati da barbari (che attualmente non ci invadono) ma nell'averci liberati dalle opere cattive, dai nostri peccati, per i quali eravamo incorsi nel dominio di satana. Poiché, quando uno viene liberato dai peccati, satana, il capo dei peccatori, non ha piú modo di dominare su di lui" ${ }^{30}$.

La vittoria di Cristo sul demonio preceduta da quella sul peccato e sulla morte, rivela tutta la sua dignità reale. L'uomo viene liberato dal dominio del demonio non mediante il prezzo pagato da Gesù al demonio, ma come il risultato dell'espiazione e della riconciliazione con $\mathrm{Dio}^{31}$. Cristo ha portato agli uomini la libertà non solo perché con la sua passione ha trionfato sul demonio ma anche perché con la dignità ha dato loro anche la libertà dei figli di Dio. L'opera redentrice di Cristo, ossia la liberazione operata da lui, è universale perchè riguarda l'intera umanità e non solo alcuni e tanto meno solo gli africani ${ }^{32}$. La

\footnotetext{
${ }^{30}$ Agostino, Espos. sul s. 84, 4 (NBA 26, p. 1217).

${ }^{31}$ Anche nell'epoca di sant'Agostino, in particolare attraverso sant'Ambrogio, si è fatto usuale il ricorso alla teoria del prezzo della liberazione. Tra le diverse affermazioni agostiniane sul sangue di Cristo versato per cancellare il debito dovuto alla liberazione degli uomini, che a volte prende forma del parlare sul prezzo ci sono anche pochi passi da cui risulta direttamente o indirettamente che anche il demonio ha ricevuto il prezzo dovuto alla liberazione. Cf. J.N.D. Kelly, Il pensiero, cit., p. 481; S. Lyonnet - L. Sabourin, Sin, Redemption, and Sacrifice. A Biblical and Patristic Study, Roma 1970, p. 208. Ciò che sembra essere l'intenzione principale di sant'Agostino quando parla del prezzo ricevuto dal demonio è la sottolineatura della rovina del demonio ossia della vittoria definitiva di Cristo. Intanto si deve tener presente che: „Bien plus, notre délivrance du démon est présentée partout comme la pure conséquence de l'expiation et de la réconciliation avec Dieu, non comme le résultat d'une rançon payée au démon. Ce principe est d'une importance capitale. Loin de mettre en première ligue je ne sais quel trafic avec le démon, partout Augustin montre que celui-ci a été vaincu précisément parce que Dieu a reçu satisfaction et a pardonné", E. Portalié, Augustin (Saint), cit., p. 2371. Cf. J. Rivière, Le dogme, cit., p. 159-178.

${ }^{32} \mathrm{Nel}$ contesto dell'esclusività in particolare quella dei Donatisti si sottolinea: „Il sangue di Cristo, ecco il prezzo. Che cosa può valere tanto? Che cosa, se non tutto l'universo? Che cosa,
} 
liberazione dell'uomo dalle schiavitù della sua natura, conseguita a prezzo cosí grande del sangue di Cristo, non esaurisce la sua misericordia ${ }^{33}$. La concezione dell'opera redentrice di Cristo di sant'Agostino, anche se nel contesto polemico fortemente incentrata sull'aspetto negativo che è proprio quello della liberazione, non si limita solo ad esso ma va ben oltre, trattando nel contesto risanatore del rinnovamento interiore e della divinizzazione dell'uomo in Cristo nell'ambito della dottrina sulla giustificazione. L'unico Figlio di Dio fatto uomo si è degnato di subire la morte per far l'uomo partecipe della vita divina e della divina misericordia. Cristo è diventato il vero fratello dell'uomo e verso suo Padre ha rivelato la sua figliolanza adottiva. Tutti i beni risultanti dall'opera redentrice di Criso, acquistati col prezzo del suo sangue, hanno il loro posto nel regno nuovo dei redenti da Cristo e nella sua Chiesa che nasce dal suo costato aperto.

L'atto redentore di Cristo ha realmente trasformato l'esistenza del credente. Anche il papa Giovanni Paolo II non smette a parlare di peccato, sapendo bene che per alcuni oggi la parola "peccato" è diventata un'espressione vuota. La redenzione di Cristo ci ha portato la redenzione dalla schiavitù del peccato. La prima verità che risulta dal fatto che Cristo è il Redentore, è che l'uomo è peccatore. Il risveglio del senso del proprio essere peccatore, della incapacità dell'uomo ad arrivare a ciò cui pure aspira in qualche sussulto ideale del cuore, aiuta molto a riscoprire la grandezza della redenzione. Diffendendo l'immaggine dell'uomo vero e la sua dignità, il papa Giovanni Paolo II come il vescovo d'Ippona parla non solo del peccato dell'uomo ma pure del suo impeto ideale e della sua sete di Infinito. Ricca è anche la riflessione di Giovanni Paolo II su ciò che rimane da fare: „possiamo e dobbiamo già fin d'ora raggiungere e manifestare al mondo la

se non l'intera umanità? Molto irriconoscenti nei confronti del prezzo sborsato per loro, ovvero enormemente superbi, sono quei tali che affermano essere stato quel prezzo cosí insignificante da potercisi ricomprare solo gli africani, ovvero si ritengono cosí importanti che ce lo volle tutto per ricomprare loro soli", Agostino, Espos. sul s. 95, 5 (NBA 27/1, p. 343). Dalla redenzione di Cristo non è escluso neanche Giuda: „Si è ricordato della sua misericordia verso Giacobbe e della sua verità a favore della casa d'Israele. Chi è questo Israele? Per non credere che si parli del solo popolo giudaico ascolta come continua: I confini della terra hanno veduto, tutti quanti, la salvezza del nostro Dio. Non si dice: «Tutta la terra»; ma tutti quanti i confini della terra. Come altrove è detto: Da un estremo all'altro della terra. Salda è l'unità di Cristo: nessuno la sminuizzi, nessuno la distrugga! Egli ha riscattato la totalità degli uomini, se cosí grande fu il prezzo che sborsò", Idem, Espos. sul s. 97, 3 (NBA 27/1, p. 407). E' frequente in sant'Agostino la sottolineatura della redenzione di tutto il mondo. Cf. Idem, Espos. sul s. 76, 17 (NBA 26, p. 991); Espos. sul s. 120, 12 (NBA 27/2, p. 1451). Cf. E. Aliaga Girbés, El sacrificio sacerdotal, cit., p. 18.

${ }^{33}$ „Io invece ho camminato nella mia innocenza; riscattami ed abbi pietà di me. Mi valga per il conseguimento della liberazione il prezzo tanto grande del sangue del mio Signore; e nei pericoli di questa vita, non mi abbandoni la tua misericordia”, Agostino, Espos. sul s. 25, I, 11 (NBA 25, p. 331). Cf. Idem, Espos. sul s. 144, 5 (NBA 28/2, p. 691-693). 
nostra unità: nell' annunciare il mistero di Cristo, nel rivelare la dimensione divina e insieme umana della Redenzione, nel lottare con instancabile perseveranza per la dignità che ogni uomo ha raggiunto e può raggiungere continuamente in Cristo. È questa la dignità della grazia dell'adozione divina ed insieme la dignità della verità interiore dell'umanità, la quale - se nella coscienza comune del mondo contemporaneo ha raggiunto un rilievo così fondamentale - ancora di più risulta per noi alla luce di quella realtà che è Lui: Gesù Cristo" (RH 11).

\section{Riassunto}

Sant'Agostino e beato Giovanni Paolo II sono le persone affascinate dal mistero di Cristo Redentore. Per capire la loro adesione a Cristo bisogna essere capaci di vedere in loro prima di tutto gli uomini di profonda fede, le persone che, come hanno scritto in diversi loro scritti, tengono il loro sguardo fisso su Gesù Cristo. Il papa Giovanni Paolo II riconoscendo nella sua Lettera apostolica Augustinum Hipponensem tutta la grandezza di sant'Agostino e chiedendogli, prima di terminare, che cosa abbia da dire agli uomini di oggi? - non solo risponde a nome suo ma anche in un certo senso diventa la sua voce, la voce di tutta la tradizione cristiana.

L'evento storico del Figlio di Dio fatto uomo, morto in croce e risorto per la salvezza degli uomini, è il vero centro della soteriologia agostiniana. Il suo concetto di redenzione è essenzialmente sacerdotale e sacrificale in quanto trova la sua definitiva espressione nel sacrificio della croce. Cristo è il vero Sacerdote fin dalla sua incarnazione, il quale non avendo peccati e non cercando altrove la vittima offre se stesso in quanto oblazione pura ed immacolata. Il suo sacrificio vero, libero e perfetto, di carattere propiziatorio, produce il perdono dei peccati (aspetto negativo) e apre la via alla riconciliazione col Padre e il rinnovamento interiore ossia la comunicazione all'uomo di una vita nuova (aspetto positivo). Se è vero che sant'Agostino dimostra con ponderatezza la dimensione morale della redenzione, tanto più è vero che mette in giusta luce tra i diversi aspetti anche quello del rapporto col demonio. Dalla croce di Cristo riluce quanto sia grande la misericordiosa disposizione di Dio per l'uomo decaduto e schiavo del peccato. La sua triplice vittoria sul peccato, sulla morte e sul demonio, riconduce l'uomo alla vita nuova nella comunione con Dio, ridonandogli la salute e lo stato di libertà. La redenzione operata da Cristo è universale, cioè compiuta a favore di tutti gli uomini, anche di Giuda. 
La riflessione di Giovanni Paolo II, almeno per quanto riguarda gli aspetti maggiormente legati al tema di Cristo Redentore dell'uomo, trova sicuramente nella Redemptor hominis, testo capitale per un' adeguata coscienza di questa verità, una delle espressioni più compiute. Cristo è l'autorivelazione compiuta di Dio. In Cristo Uomo nuovo e definitivo avviene la chiamata dell'uomo a partecipare alla vita di Dio, non in modo formale, ma sostanziale perché ratificata nella effusione dello Spirito. Cristo è il Redentore dell'uomo in quanto ricrea l'umanità. In Cristo il Dio della creazione si manifesta come il Dio della redenzione facendo sì che l'Alleanza sia compiuta. In Cristo viene manifestato in modo nuovo e mirabile che la redenzione non consiste soltanto nella remissione dei peccati dell'uomo, ma in essa si esprime, secondo una propria logica, il compimento del piano originale di Dio. In Cristo l'uomo e il mondo ritrovano il contatto con Dio. Gesù Cristo è la rivelazione definitiva dell'amore di Dio che è più grande del peccato; è amore sempre pronto a sostenere l'uomo e a perdonarlo. Per Giovanni Paolo II, infatti, la redenzione apportata da Cristo è universale, non solo nel senso che è sovrabbondante per tutto il genere umano ed è proposta a ciascuno dei suoi membri in particolare, ma anche nel senso che è applicata di fatto a tutti gli uomini presi individualmente.

\section{Bibliography}

Agostino, Esposizioni sui Salmi (Enarrationes in Psalmos), in: Opera Omnia di Sant'Agostino (Nuova Biblioteca Agostiniana), vol. 25-28/2, Roma 1982², 1990², $1993^{2}$, ed. Città Nuova.

Agostino, Commento alla Prima Epistola di S. Giovanni, in: Opera Omnia di Sant'Agostino (Nuova Biblioteca Agostiniana), vol. 24/2, Roma 1985², ed. Città Nuova, p. 1014-2080.

Altaner B., Patrologia, Torino 1952, ed. Marietti.

Bonner G., The desir for God and the need for grace in augustine's theology, in: Congresso Internazionale su s. Agostino nel XVI centenario della conversione, (Roma 15-20 sett. 1986), vol. I, Roma 1987, ed. Institutum Patristicum Augustinianum, p. 199-214.

Bordoni M., Gesù di Nazaret, Signore e Cristo, vol. III. Il Cristo annunciatore della Chiesa, Roma 1982, ed. Herder.

Boyer Ch., Le triple amour du Christ pour les hommes dans les écrits de Saint Augustin, in: Cor Jesu, Commentationes in litteras Encyclicas Pii PP. XII "Haurietis Aquas", ed. A. Bea - H. Rahner, vol. I, Roma 1959, ed. Herder, p. 571-594.

Cipriani N., Gesù il Figlio. Testi dei Padri della Chiesa, Roma 1997, ed. Città Nuova.

Giovanni Paolo II, Lettera apostolica Augustinum Hipponensem del Sommo Pontefice Giovanni Paolo II nel XVI centenario della conversione di S. Agostino (28.08.1989), 
in: R. Piccolomini, N. Monopoli, L'attualità di Agostino. Commento alla Lettera apostolica Agostino d'Ippona di Giovanni Paolo II, Roma 1986, ed. Città Nuova, p. 213-249 [testo orig. in: AAS 79 (1987) 137-170].

Giovanni Paolo II, Lettera enciclica Redemptor hominis (4 marzo 1979), Bologna 1979, ed. Dehoniane Bologna [testo orig. in: AAS 71 (1979) 257-324].

Jossua J.-P., Le salut, Incarnation ou Mystère Pascal. (Chez les Pères de l'Église de saint Irénée à saint Léon le Grand), Paris 1968, ed. Cerf.

Kelly J.N.D., Il pensiero delle origini, Bologna 1972, ed. Il Mulino.

Lyonnet S. - Sabourin L., Sin, Redemption, and Sacrifice. A Biblical and Patristic Study, Roma 1970, ed. Biblical Institute Press.

Madec G., Christus, in: Augustinus-Lexikon, ed. C. Mayer, vol. I, Basel 1986, ed. Schwabe \& Co., p. 845-907.

Mondin B., Dio: Chi è? Elementi di teologia filosofica, Milano 1990, ed. Massimo.

Remy G., Le Christ mediateur dans l'oeuvre de Saint Augustin, Lille-Paris 1979, ed. Champion.

Rivière J., Le dogme de la rédemption chez saint Augustin, Paris 1933, ed. Lecoffre.

Sanguinetti Montero A., Gratuidad y respuesta del hombre a Dios. Estudio en las "Enarrationes in Psalmos" de san Agustín, Montevideo 1983, ed. Instituto Teológico del Uruguay Monseñor Mariano Soler.

Scano E., Il Cristocentrismo e i suoi fondamenti dogmatici in s. Agostino, Torino 1951, ed. L.I.C.E.: R. Berruti e Co.

Sesboüé B., Gesù Cristo l'unico mediatore, Cinisello Balsamo 1991, ed. Paoline.

Spada D., La misericordia nel pensiero di san Tommaso d'Aquino, Dives in misericordia. Commento all'enciclica di Giovanni Paolo II, Roma 1981, ed. J. Saraiva Martines.

Studer B., Dio Salvatore nei Padri della Chiesa, Roma 1986, ed. Borla.

Turner H.E.W., Jésus le Sauveur. Essai sur la doctrine patristique de la Rédemption, Paris 1965, ed. Cerf.

Vassallo A., Inquietum cor. Con Agostino alla ricerca di Dio, Palermo 1988, ed. Augustinus.

Viciano A., Titoli soterici di Cristo nei "Sermones" di sant'Agostino, in: Congresso Internazionale su s. Agostino nel XVI centenario della conversione, (Roma 15-20 sett. 1986), vol. II, Roma 1987, ed. Institutum Patristicum Augustinianum, p. 323-335.

Vincent M., Saint Augustine, maître de prière d'après les Enarrationes in Psalmos, Paris 1990, ed. Beauchesne. 